\title{
Global Stability of Bilinear Reinforced Slopes
}

\author{
Xiaobo Ruan • Dov Leshchinsky • \\ Ben A. Leshchinsky
}

Accepted: 3 October 2014 / Published online: 16 October 2014

(C) Springer New York 2014

\begin{abstract}
Design and construction of geosynthetic reinforced simple slopes are a common practice. These types of slope commonly use a single inclination, termed a linear slope. Design of linear slopes is frequently done using limit equilibrium (LE) analysis. The scenario of two tiered slopes, one with a vertical upper tier and another with an inclined lower tier, is termed in this study as a bilinear slope. It increases the right-of-way for various types of infrastructure in the same way as linear slopes. This paper presents a LE approach to analyze such bilinear reinforced slopes. This LE analysis uses a top-down log spiral mechanism and is rigorous in the sense that it satisfies equilibrium at the limit state. The presented formulation and numerical scheme yield the required, unfactored reinforcement strength. Results are presented in the form of stability charts, enabling quick assessment of reinforcement strength required for stability. A complementary chart shows the quantity of backfill saved when using bilinear reinforced slope versus the alternative, equivalent linear reinforced slope. A shallow inclination of the lower tier eliminates the need for its reinforcements although it is surcharged by a vertically reinforced slope. That is, the reinforcement in the upper tier also resists failures through its foundation, an aspect that is considered in the analysis. However, if the lower tier is steep, it may require some reinforcement as the
\end{abstract}

\footnotetext{
X. Ruan • D. Leshchinsky $(\bowtie)$

Department of Civil and Environmental Engineering, University of Delaware, Newark, DE 19716, USA e-mail: dov@udel.edu

X. Ruan

e-mail: xiaoboruan@163.com

X. Ruan

College of Civil and Transportation Engineering, Hohai University, Nanjing 210098, China

D. Leshchinsky

ADAMA Engineering, Inc., P.O. Box 7838, Newark, DE 19714, USA

B. A. Leshchinsky

Department of Forest Engineering, Resources and Management, Oregon State University, Corvallis, OR 97331, USA

e-mail: ben.leshchinsky@oregonstate.edu
} 
resistance of the geosynthetics placed in the upper tier is not sufficient for adequate stability.

Keywords Bilinear slope $\cdot$ Reinforced soil $\cdot$ Geosynthetics $\cdot$ Limit equilibrium

\section{Introduction}

Right-of-way for structures is often constructed using slopes. Space and/or aesthetics sometimes require steep slopes, which are typically stable due to inclusion of reinforcements, such as geosynthetics. Typically, reinforced steep slopes (RSS) have a single inclination, i.e., simple steep slopes. However, considerations such as economics (e.g., less select reinforced backfill), surface drainage (e.g., faster removal of water), and aesthetics, may justify bilinear slope angles which render the same right-of-way as a simple steep slope.

In the context of this study, a wall is defined as a slope with zero batter (i.e., vertical) while a "slope" has a batter greater than zero (non-vertical). When reinforced walls are placed over reinforced slopes, a bilinear slope angle is produced, yielding the same right-of-way as a single equivalent, yet less steep slope. The objective of this study is to conduct a rigorous limit equilibrium (LE) analysis exploring the impact of such bilinear slopes on the maximum mobilized reinforcement force required for stability. It is assumed that the foundation soil is competent (i.e., will not allow development of shear throughout its continuum). It is noted that Leshchinsky and Han [1] compared the stability of tiered reinforced walls calculated using LE and Finite Difference Analyses. This comparison exhibited good agreement indicating that, theoretically, both approaches are reasonable. Furthermore, FHWA [2] provide guidelines for design using LE analysis. Hence, the outcome of this work provides an insight into a practical use of reinforcement in bilinear slopes.

The publication rate on reinforced tiered walls has increased since 2000. These publications include field studies, centrifugal modeling, numerical analysis, and LE analysis [1, 3-5]. A comprehensive literature review is presented by Mohamed, Yang, and Hung (2013) [5] who have also verified the validity of LE analysis in terms of fining the maximum load in the reinforcement. It appears that much of the literature relates to reinforced tiered walls, i.e., not reinforced walls over reinforced slopes as is the case in this paper. Leshchinsky (1997) [6] suggested a methodology for tiered slopes/walls following a computerized procedure he introduced in 1991 using his program StrataSlope later modified to program ReSlope. This was a top-down approach where the upper reinforced tier is first designed followed with the design of the tiers below considering the upper tiers as surcharge. However, the Leshchinsky (1997) [6] approach does not replicate the current approach of bilinear slope as it assumed an offset between tiers.

\section{Formulation}

The outlined analysis assumes log spiral slip surfaces as part of the limit equilibrium (LE) formulation with a numerical procedure implemented in MATLAB, ver. 8.2 
(R2013b) [7] software - refer to Fig. 1 for notation and convention. The soil in the reinforced zone is considered as a homogenous and cohesionless material. Furthermore, the reinforcement force that holds the system stable is calculated at the intersection with the log spiral slip surface is considered horizontal. It is further assumed that all layers of reinforcements are long enough to render the critical slip surface passing through all layers without exceeding pullout resistance. It is noted that front-end pullout could be exceeded if the connection strength to the facing is insufficient. This aspect is discussed by Leshchinsky et al. (2014) [8]. This reference shows that for stability purposes, the required "connection" strength is a fraction of the maximum load in the reinforcement. Finally, it is assumed that the slip surface extends between the crest and either the toe of the upper tier (i.e., point $E$ at the base of the "wall") or at the toe of the lower tier (i.e., point $O$ at the base of the "slope"). It is noted that the failure mass (i.e., Region $O B C E$ in Fig. 1) includes the wall facing, thus rendering the internal soil-facing irrelevant to the global analysis, implicitly assuming that the unit weight of facing is approximately the same as that of the reinforced soil.

In a log spiral analysis, the moment LE equation can be explicitly stated (i.e., without resorting to statical assumptions). However, using moment LE to maximize the required reinforcement load implicitly satisfies the LE equilibrium equations as well [9]. Hence, all equations of limit equilibrium are satisfied, justifying the classification of the defined analysis as rigorous. For brevity, only the necessary equations are presented here. However, for the stated formulation and applications, one may be referred to additional literature [10-13] to realize the details of the methodology of using the moment LE equation resulting from postulated log spiral mechanism. It is noted that log spiral stability analysis is common in homogeneous slopes [14].

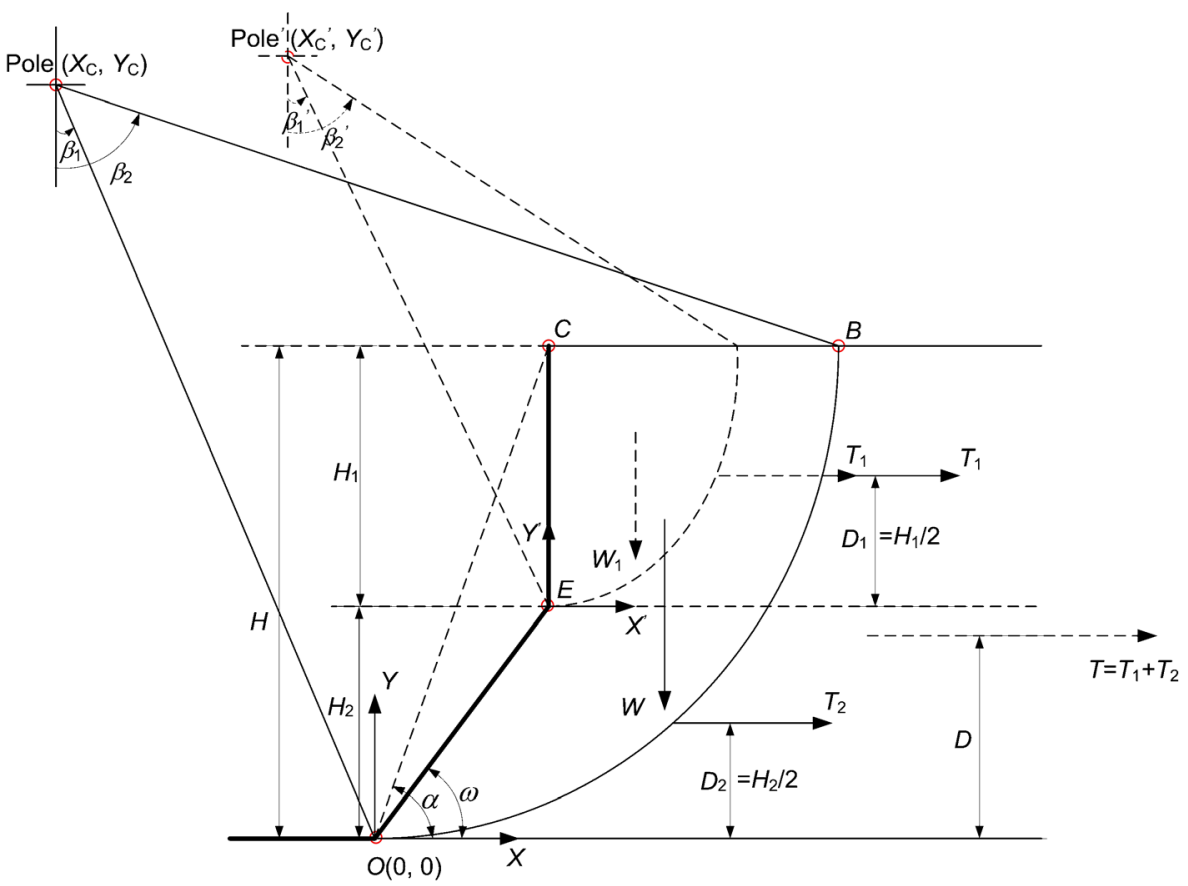

Fig. 1 Notation and convention for the presented LE approach 
Analysis

In Fig. 1, the resisting or slide-restraining forces, $T_{1}$ and $T_{2}$, are the resultant force of all reinforcement layers for the upper tier (wall) and the lower tier (slope), respectively. Stated differently, $T_{1}$ and $T_{2}$ are the summation the maximum mobilized force in all layers of reinforcement, $\sum T_{\max }$, in the upper tier and in lower tier, respectively. The driving force, $W$, is the weight of the entire failure mass. The total required reinforcement force, $T$, is the sum of $T_{1}$ and $T_{2}$. The line of action of $T_{1}, D_{1}$, is measured from the bottom of the upper tier while the lines of action of $T_{2}$ and $T, D_{2}$, and $D$, are both measured from the bottom of the lower tier.

The location of the resultant force of the reinforcements is not known and must be assumed. Upon parametric studies, one can realize the impact of such an assumption on the magnitude of the maximum required reinforcement force or the location of the critical slip surface. For the current design guidelines of Mechanically Stabilized Earth (MSE) walls [2, 15, 16], for a horizontal and surcharge-free crest subjected to static conditions, the height of the resultant is one third of the height of the wall. With assumed surcharge or seismicity, the elevation of resultant goes up. Therefore, it is reasonable to assume $D_{1}$ and $D_{2}$, to act at $H_{1} / 2$ and $H_{2} / 2$, respectively; augmenting an assumed variable $D$ calculated through moment equilibrium equation $T$

$$
D=\left(M_{T 1}+M_{T 2}\right) / T
$$

where $T=T_{1}+T_{2}=\sum T_{\max }$ (the summation of maximum forces in all layers from $O$ to $C$ ) and $M_{\mathrm{T} 1}$ and $M_{\mathrm{T} 2}$ are moments due to $T_{1}$ and $T_{2}$, respectively. Note that moments are calculated about the pole of the log spiral-Fig. 1 . The resisting moments, $M_{\mathrm{T} 1}$ and $M_{\mathrm{T} 2}$, can be determined using $T_{1}$ and $T_{2}$ multiplied by their respective leverage arms. Similarly, the driving moment about the pole, $M_{\mathrm{W}}$, can be calculated using $W$ multiplied by its corresponding leverage arm. The weight of the sliding mass, $W$, is defined by the analyzed log spiral.

For completeness, the expression for the resultant resisting force in the upper tier, $T_{1}$, is reproduced here from Leshchinsky, et al. [9]

$$
\begin{aligned}
T_{1}= & {\left[\gamma_{\mathrm{d}} \int_{\beta_{1}^{\prime}}^{\beta_{2}^{\prime}}\left(A^{\prime} e^{-\psi \beta^{\prime}} \cos \beta^{\prime}-A^{\prime} e^{-\psi \beta_{2}^{\prime}} \cos \beta_{2}^{\prime}\right)\left(A^{\prime} e^{-\psi \beta^{\prime}} \sin \beta^{\prime}\right)\left(A^{\prime} e^{-\psi \beta^{\prime}}\right)\left(\cos \beta^{\prime}-\psi \sin \beta^{\prime}\right) d \beta^{\prime}\right] } \\
& /\left(A^{\prime} e^{-\psi \beta_{1}^{\prime}} \cos \beta_{1}^{\prime}-D_{1}\right)
\end{aligned}
$$

where $\gamma_{\mathrm{d}}$ is the unit weight of the reinforced soil; $\beta_{1}^{\prime}$ and $\beta_{2}^{\prime}$ are angles at points where the $\log$ spiral slip surface enters and exits the upper tier; $\beta^{\prime}$ is the angle in polar coordinates defined relative to the Cartesian coordinate system translated to Pole' $\left(X_{\mathrm{C}}^{\prime}, Y_{\mathrm{C}}^{\prime}\right)$ from the origin $E$-Fig. 1 ; and $A^{\prime}$ is $\log$ spiral constant, i.e., $H_{1} /$ $\left[\exp \left(-\psi \beta_{1}^{\prime}\right) \cos \beta_{1}^{\prime}-\exp \left(-\psi \beta_{2}^{\prime}\right) \cos \beta_{2}^{\prime}\right]$, where, $H_{1}$ is height of the upper tier, $\psi=\tan \phi_{\mathrm{d}}$, and $\phi_{\mathrm{d}}$ is the design internal angle of friction.

The resistive moment component due to normal and shear stress distributions along the log spiral at a LE state vanishes since its elemental resultant force goes through the 
pole. Consequently, at a LE state, the resisting and driving moments are equal as shown:

$$
M_{\mathrm{W}}=M_{\mathrm{T} 1}+M_{\mathrm{T} 2}
$$

where

$$
\begin{gathered}
M_{\mathrm{W}}=\gamma_{\mathrm{d}} \int_{\beta_{1}}^{\beta_{2}}\left(\mathrm{Ae}^{-\psi \beta} \cos \beta-\mathrm{Ae}^{-\psi \beta_{2}} \cos \beta_{2}\right)\left(\mathrm{Ae}^{-\psi \beta} \sin \beta\right)\left(\mathrm{Ae}^{-\psi \beta}\right)(\cos \beta-\psi \sin \beta) d \beta \\
-\left(\gamma_{\mathrm{d}} \times H_{1} H \cot \alpha\right)\left(\mathrm{Ae}^{-\psi \beta_{1}} \sin \beta_{1}+H \cot \alpha / 2\right)-\left(\gamma_{\mathrm{d}} H_{2} H \cot \alpha / 2\right)\left(\mathrm{Ae}^{-\psi \beta_{1}} \sin \beta_{1}+H \cot \alpha / 3\right) \\
M_{\mathrm{T} 1}=T_{1}\left(\mathrm{Ae}^{-\psi \beta_{1}} \cos \beta_{1}-H_{2}-D_{1}\right) \\
M_{\mathrm{T} 2}=T_{2}\left(\mathrm{Ae}^{-\psi \beta_{1}} \cos \beta_{1}-D_{2}\right)
\end{gathered}
$$

Using Eqs. 2, 3, and 3(a-c), one can solve $T_{2}$ which is as follows:

$$
\begin{aligned}
T_{2}= & {\left[\gamma_{\mathrm{d}} \int_{\beta_{1}}^{\beta_{2}}\left(\mathrm{Ae}^{-\psi \beta} \cos \beta-\mathrm{Ae}^{-\psi \beta_{2}} \cos \beta_{2}\right)\left(\mathrm{Ae}^{-\psi \beta} \sin \beta\right)\left(\mathrm{Ae}^{-\psi \beta}\right)(\cos \beta-\psi \sin \beta) d \beta\right.} \\
& -\left(\gamma_{\mathrm{d}} H_{1} H \cot \alpha\right)\left(\mathrm{Ae}^{-\psi \beta_{1}} \sin \beta_{1}+H \cot \alpha / 2\right)-\left(\gamma_{\mathrm{d}} H_{2} H \cot \alpha / 2\right)\left(\mathrm{Ae}^{-\psi \beta_{1}} \sin \beta_{1}+H\right. \\
& \left.\times \cot \alpha / 3)-T_{1}\left(\mathrm{Ae}^{-\psi \beta_{1}} \cos \beta_{1}-H_{2}-D_{1}\right)\right] /\left(\mathrm{Ae}^{-\psi \beta_{1}} \cos \beta_{1}-D_{2}\right)
\end{aligned}
$$

where $H_{2}$ and $H$ are heights of the lower tier and the bilinear slope, respectively; $\beta_{1}$ and $\beta_{2}$ are angles of points where the log spiral enters and exits the bilinear slope-Fig. $1 ; \alpha$ is the angle of the bilinear slope; $\beta$ is the angle in polar coordinates defined relative to the Cartesian coordinate system translated to Pole $\left(X_{\mathrm{C}}, Y_{\mathrm{C}}\right)$ from the origin $O(0,0)$; and $A$ is $\log$ spiral constant, i.e., $H /\left[\exp \left(-\psi \beta_{1}\right) \cos \beta_{1}-\exp \left(-\psi \beta_{2}\right) \cos \beta_{2}\right]$.

For a dimensionless analysis using $T_{1}$ and $T_{2}$, one can, respectively define $K_{\mathrm{T} 1}$ and $K_{\mathrm{T} 2}$ as

$$
\begin{aligned}
K_{\mathrm{T} 1} & =\frac{T_{1}}{0.5 \gamma_{\mathrm{d}} H^{2}} \\
K_{\mathrm{T} 2} & =\frac{T_{2}}{0.5 \gamma_{\mathrm{d}} H^{2}} .
\end{aligned}
$$

\section{Numerical Procedure}

The numerical procedure for calculating the maximum value of $T_{2}$ was achieved by using MATLAB, ver. 8.2 (R2013b) [7] software. Figure 2 shows the computational scheme used to determine the maximization of $T_{2}$. 
Fig. 2 Computational scheme for maximization of $T_{2}$

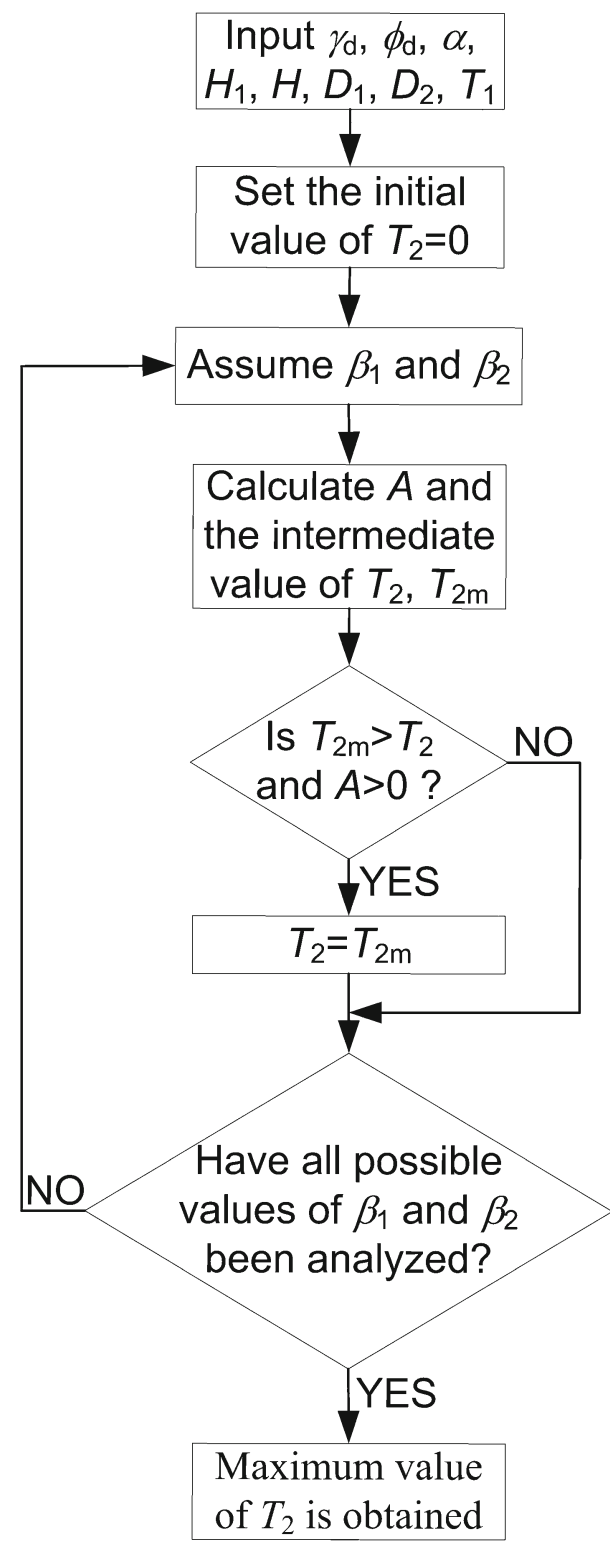

\section{Results}

For a meaningful presentation of results, a parameter $\lambda$ is introduced, defined as $H_{1} / H$. When $\lambda=0$, this case corresponds to $H_{1}=0$, that is, the bilinear slope is equivalent to the problem having an equivalent slope inclination of $\alpha$. When $\lambda$ approaches $1, H_{2}$ approaches zero, implying that the bilinear slope degenerates to a vertical MSE wall. These two limits bracket the bounds of the reinforced bilinear slope, whose stability and required reinforcement strength is the primary objective of this work. 
Stability Charts

Figure $3 \mathrm{a}-\mathrm{e}$ shows that $K_{\mathrm{T} 2}$ decreases as $\lambda$ increases for different values of $\alpha . K_{\mathrm{T} 1}$ increases with an increase in $\lambda$. Each chart is drawn for a selected $\phi_{\mathrm{d}}$. Note that the values of $34^{\circ}$ and $40^{\circ}$ are AASHTO's allowable default and maximum design values for the internal angle of friction for the selected backfill [15].

The stability charts (see Fig. 3a-e) may assist in design of bilinear slopes in consideration of internal stability. Presented at the end of this study is an example, which demonstrates the application of these design charts.

\section{Reduced Backfill Volume}

One potential economic advantage of using a bilinear slope is a reduced volume of backfill needed to produce the required right-of-way. The reduced volume $S$, namely the area of triangle $O C E$, per unit length of the slope, can be calculated from the difference in geometries as a function of $\lambda$ and $\alpha$. Its normalized value per unit length can be represented by $V_{\mathrm{U}}=\left(S / H^{2}\right.$-Fig. 4).

\section{Critical Slip Surfaces}

In Fig. 5a-c, traces of critical slip surfaces are presented for different values of $\lambda$ while $\alpha$ is equal to $60^{\circ}$ and $\phi_{\mathrm{d}}$ are equal to $30^{\circ}, 34^{\circ}$, and $40^{\circ}$. It is noted that "critical slip surfaces" means that these surfaces produce maximum $T_{1}$ and $T_{2}$ forces; hence, they govern design. From these figures, when $\lambda$ or $\phi_{\mathrm{d}}$ approaches a certain value, the critical slip surface for the entire bilinear slope emerges at the toe of the upper tier rather than at the toe of the lower tier. In such a case, the value of $T_{2}$ equals zero, implying that the lower tier is shallow enough to not require reinforcements to support the surcharge of the upper tier.

\section{Line of Action of T}

Recall that $D$, the location of the resultant reinforcement force $T$, is a result of assumed line of action of $T_{1}$ and $T_{2}$. That is, $D$ is the weighted average $D_{1}$ and $D_{2}$ considering $T_{1}$ and $T_{2}$. For selected values of $\phi_{\mathrm{d}}$, Fig. 6a-c shows the normalized value of $D,(D / H)$ as a function of $\lambda$ for different values of $\alpha$. In these figures, the $(D / H)$ ratio initially decreases as $\lambda$ increases, followed by an increase and subsequent linear decrease. When the $(D / H)$ ratio approaches a maximum value, $T$ becomes equal to $T_{1}$ (i.e., $T_{2}=0$ meaning that the critical slip surface emerges at the toe of the upper tier).

\section{Example}

The following example demonstrates how application of the presented stability charts. Consider the problem shown in Fig. 7 where $\gamma_{\mathrm{d}}=20 \mathrm{kN} / \mathrm{m}^{3}, \phi_{\mathrm{d}}=34^{\circ}, H_{1}=4.8 \mathrm{~m}, H=$ $8.4 \mathrm{~m}$, and $\alpha=70^{\circ}$, angle of the lower tier is $\omega=49.7^{\circ}$, and vertical spacing between reinforcement layers is $S_{\mathrm{v}}=0.6 \mathrm{~m}$. 


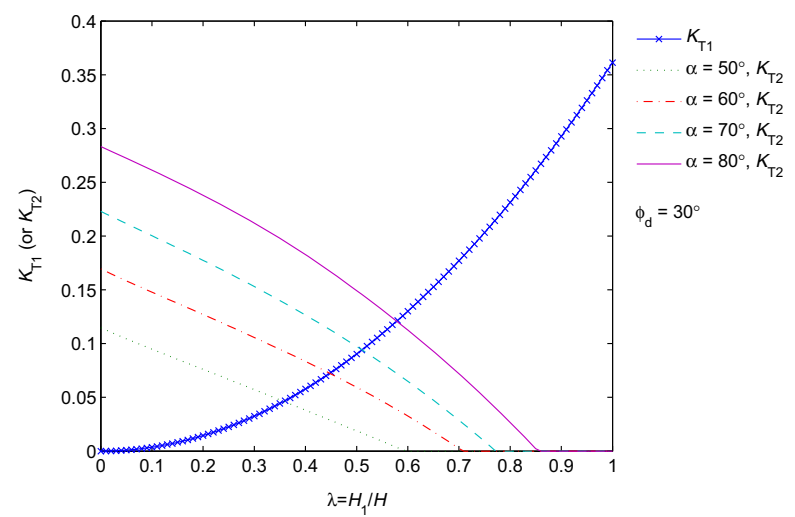

(a)

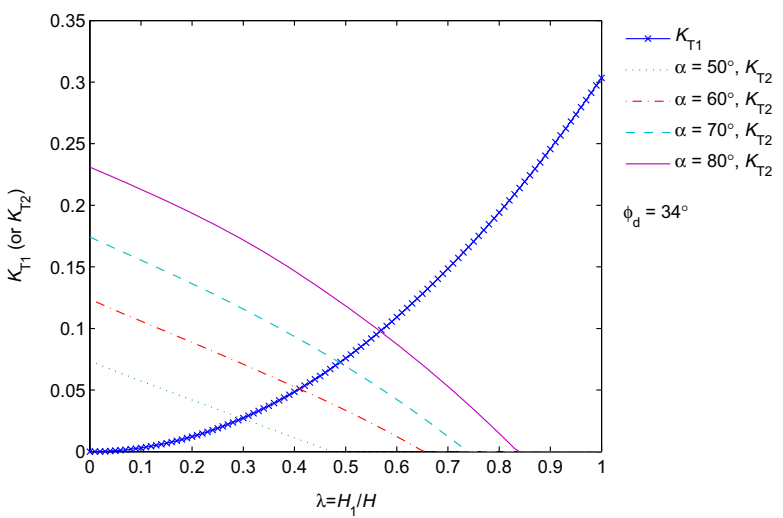

(b)

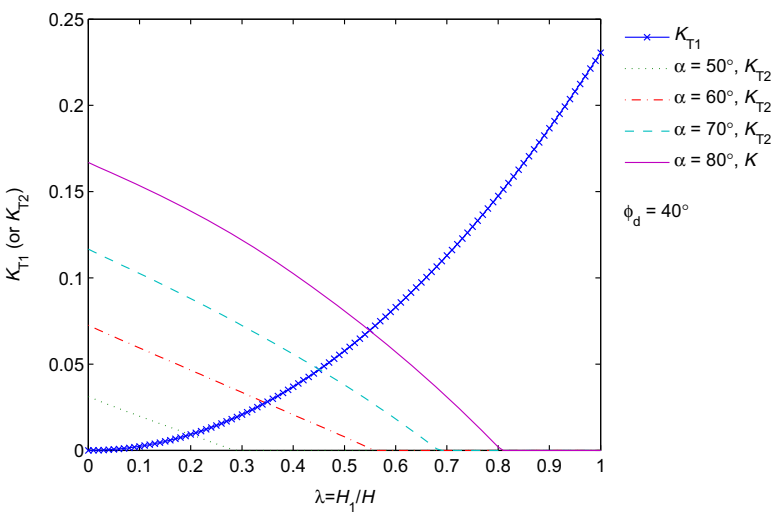

(c)

Fig. 3 Stability charts for different $\alpha$ : a $\phi_{\mathrm{d}}=30^{\circ}, \mathbf{b} \phi_{\mathrm{d}}=34^{\circ}, \mathbf{c} \phi_{\mathrm{d}}=40^{\circ}, \mathbf{d} \phi_{\mathrm{d}}=45^{\circ}$, and e $\phi_{\mathrm{d}}=50^{\circ}$

From Fig. 3b, using $\lambda=4.8 / 8.4=0.57$, one can get $K_{\mathrm{T} 1}=0.10$, and $K_{\mathrm{T} 2}=0.05 . T_{1}$ and $T_{2}$ can now be calculated from Eqs. 5 and 6 , respectively, as $T_{1}=70.6 \mathrm{kN} / \mathrm{m}$ and $T_{2}=$ $35.3 \mathrm{kN} / \mathrm{m}$. Selecting $D_{1}=H_{1} / 2$ and $D_{2}=H_{2} / 2$ usually corresponds to a uniform 


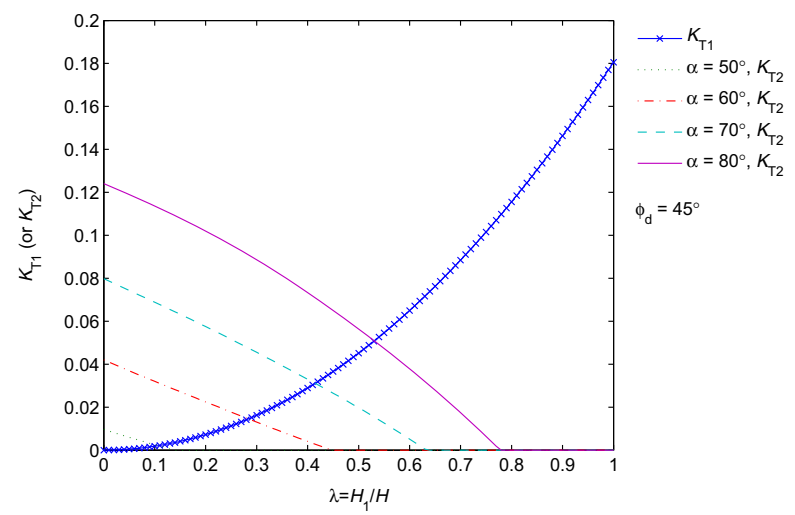

(d)

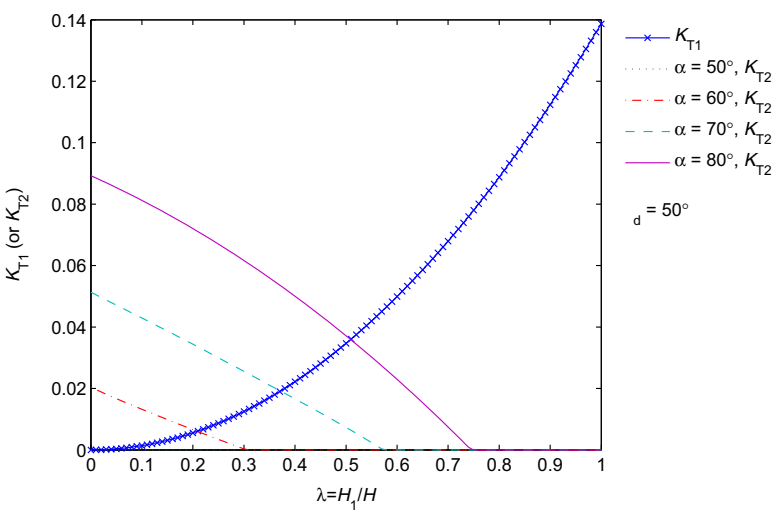

(e)

Fig. 3 (continued)

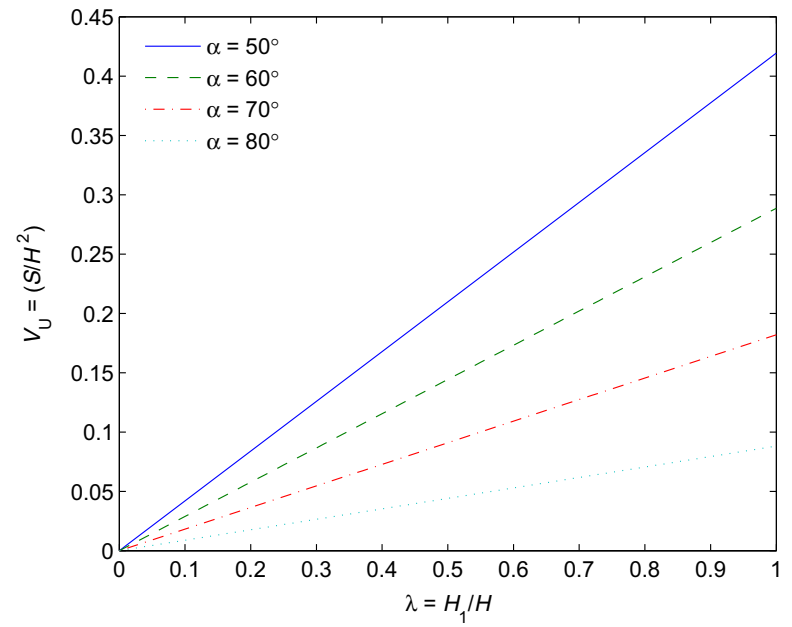

Fig. $4 V_{\mathrm{U}}$ as function of $\lambda$ and $\alpha$

\section{Springer}


Fig. 5 Critical slip surface for $\alpha=60^{\circ}$ : a $\lambda=0.6, \mathbf{b} \lambda=0.7$, and $\mathbf{c}$ $\lambda=0.8$

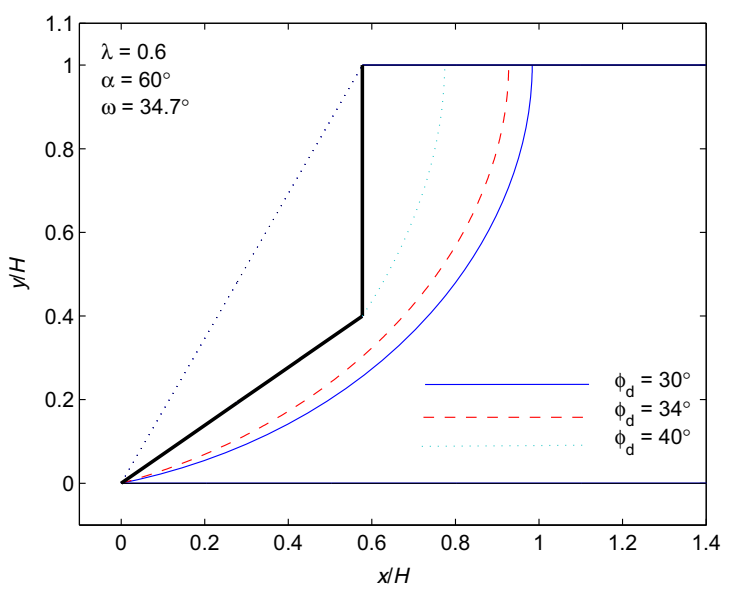

(a)

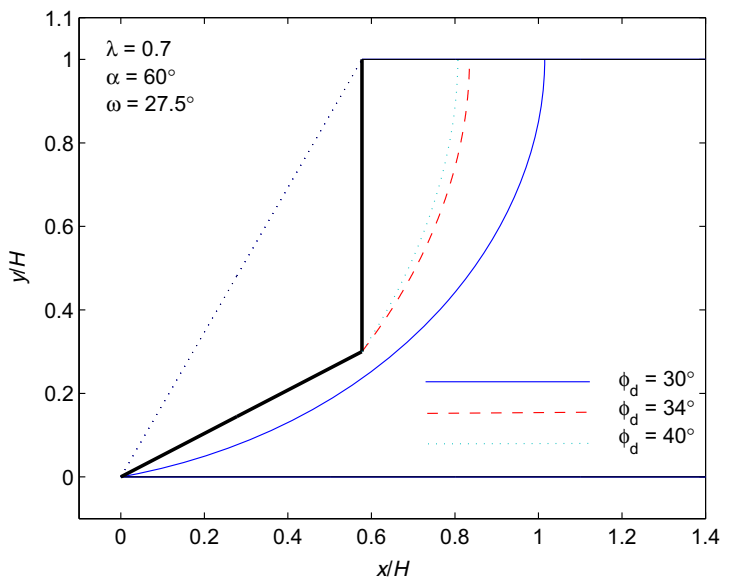

(b)

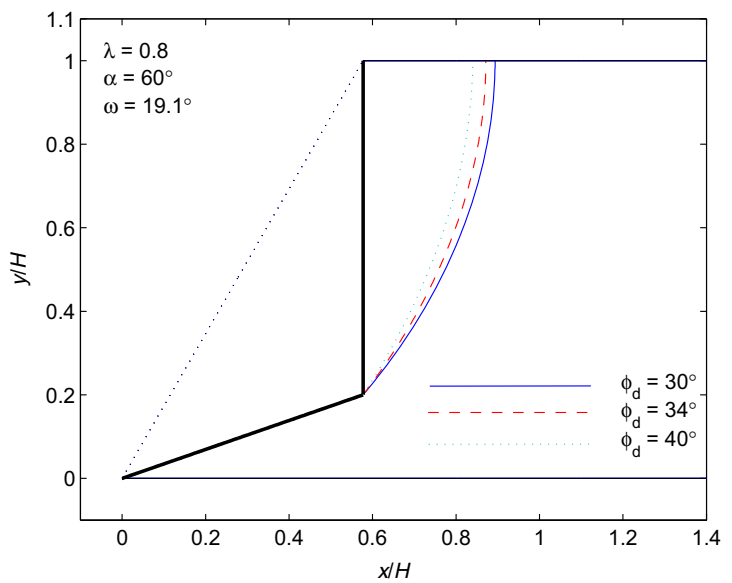

(c) 
Fig. $6 D / H$ versus $\lambda$ for different $\alpha$ : a $\phi_{\mathrm{d}}=30^{\circ}, \mathbf{b} \phi_{\mathrm{d}}=34^{\circ}$, and c $\phi_{\mathrm{d}}=40^{\circ}$

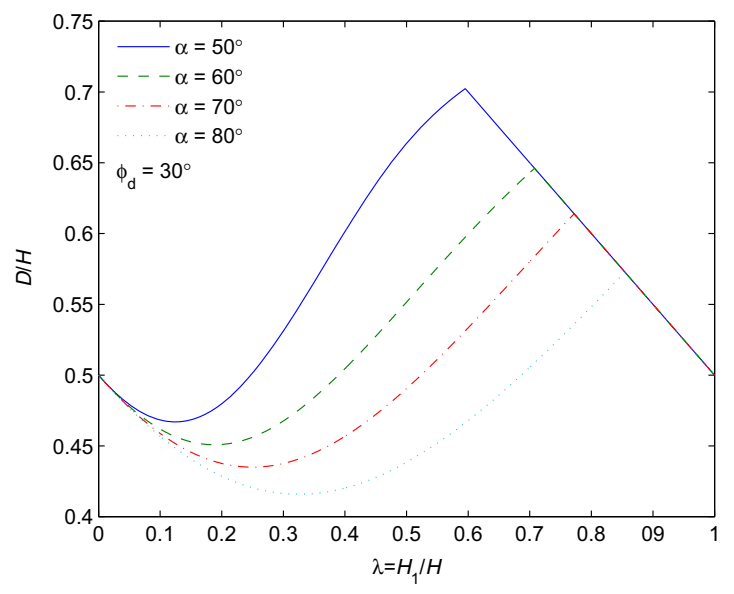

(a)

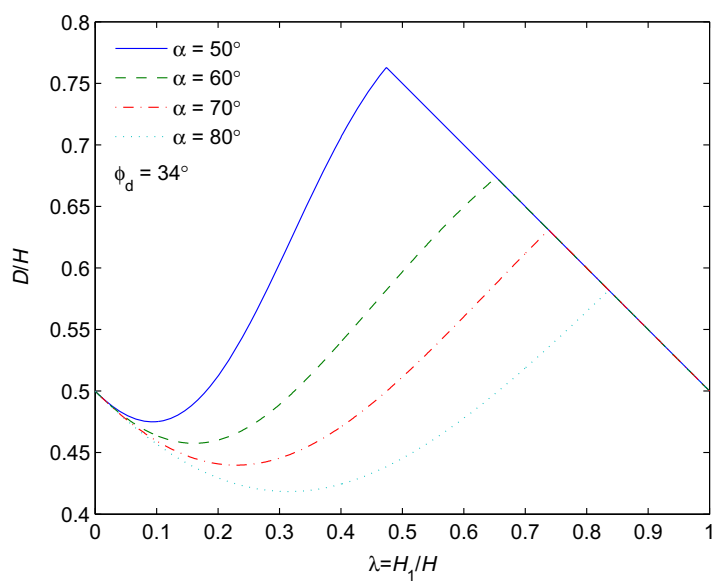

(b)

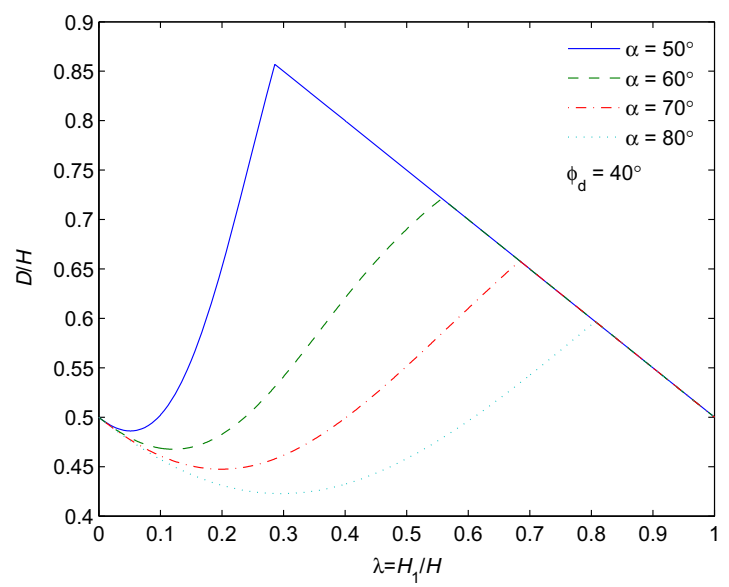

(c) 


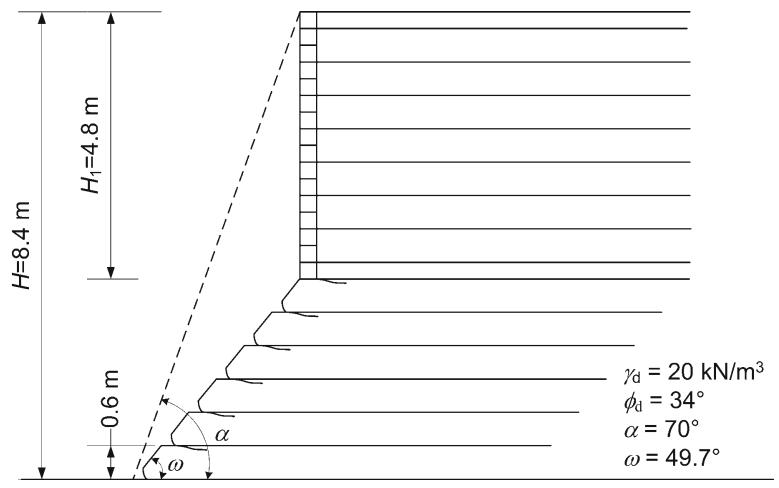

Fig. 7 Details of example problem

distribution of maximum loading throughout all layers. Such a distribution is common in reinforced slope stability calculations. Subsequently, $T_{\max }$ for the upper tier, $T_{1 \max }$, is $T_{1} / n_{1}$ and for the lower tier, $T_{2 \max }$, is $T_{2} / n_{2}$ where $n_{1}$ and $n_{2}$ are the number of layers in the upper and lower tiers, respectively. Thus, the uniform maximum reinforcement load in the upper tier is $T_{1 \max }=8.8 \mathrm{kN} / \mathrm{m}$ and in the lower tier is $T_{2 \max }=5.9 \mathrm{kN} / \mathrm{m}$.

However, when the bilinear slope degenerates to an equivalent slope with the inclination of $70^{\circ}$ (i.e., $\lambda=0$ ), one can get $K_{\mathrm{T} 1}=0$, and $K_{\mathrm{T} 2}=0.17$ from Fig. 3b. The $T_{\max }$ for the equivalent slope is $T_{2} /\left(n_{1}+n_{2}\right)$. Therefore, $K_{\mathrm{T} 2}=0.17$ yields $T_{\max }=8.6 \mathrm{kN} /$ $\mathrm{m}$ assuming a uniform distribution of maximum reinforcement load as commonly done in global stability.

For field installation, the reinforcement strength is selected based on the maximum expected reinforcement tensile load $\left(T_{\max }\right)$. For the equivalent $70^{\circ}$ slope, the selected strength will be based upon the aforementioned $8.6 \mathrm{kN} / \mathrm{m}$ whereas for the bilinear slope, it will be $8.8 \mathrm{kN} / \mathrm{m}$ for the $4.8 \mathrm{~m}$ high upper tier ("wall") and $5.9 \mathrm{kN} / \mathrm{m}$ for the $3.6 \mathrm{~m}$ tall lower tier ("slope"). For this case, the difference in required strength due to bilinear slope is minor in the upper tier and moderate in the lower tier if one is to use there a weaker reinforcement. There is a saving in backfill volume as well as change in surface drainage characteristics.

\section{Concluding Remarks}

Presented is a limit equilibrium approach to analyze bilinear reinforced slopes. The LE analysis uses a top-down log spiral mechanism and is rigorous in the sense that it satisfies limit state equilibrium. The formulation is described and when combined with the presented numerical scheme, it will result in the required unfactored reinforcement strength for design of bilinear slopes. More results are presented in the form of a series of stability charts, which facilitate assessment of required reinforcement strength required for stable design. A complementary chart shows the reduced amount of backfill needed when using bilinear reinforced slope versus the alternative equivalent, linear reinforced slope.

When the inclination of the lower tier gets shallower, no reinforcement is needed, although it is surcharged by a vertically reinforced slope (i.e., "wall"). This is due to the 
reinforcement in the upper tier, which resists failures through its foundation. While the change in required strength of reinforcements due to a bilinear slope may not be substantial, there is a reduction in required backfill material.

Acknowledgments The China Scholarship Council (CSC) granted to the first author, enabling him to be at $\mathrm{UD}$, is greatly appreciated.

\section{References}

1. Leshchinsky, D., Han, J.: Geosynthetic reinforced multitiered walls. J. Geotech. Geoenviron. Eng. 130(12), 1225-1235 (2004)

2. Federal Highway Association (FHWA): Design and construction of mechanically stabilized earth walls and reinforced soil slopes, FHWA-NHI-10-024, Berg, R.R., Christopher, B.R., Samtani, N.C., eds. Washington, DC (2009)

3. Yoo, C., Jung, H.S.: Measured behavior of a geosynthetic reinforced segmental retaining wall in a tiered configuration. Geotext. Geomembr. 22(5), 359-376 (2004)

4. Yoo, C., Kim, S.B.: Performance of a two-tier geosynthetic reinforced segmental retaining wall under a surcharge load: full-scale load test and 3D finite element analysis. Geotext. Geomembr. 26(6), 447-518 (2008)

5. Mohamed, S.B.A., Yang, K.H., Hung, W.Y.: Limit equilibrium analyses of geosynthetic-reinforced twotiered walls: calibration from centrifuge tests. Geotext. Geomembr. 41, 1-16 (2013)

6. Leshchinsky, D.: Design procedure for geosynthetic reinforced steep slopes, report REMR-GT-23, geotechnical laboratory. US Army Corps of Eng., Waterways Experiment Station, Vicksburg (1997)

7. MATLAB, ver. 8.2 (R2013b): The MathWorks, Inc., 3 Apple Hill Drive, Natick, MA 01760-2098 (2013)

8. Leshchinsky, D., Kang, B.J., Han, J., Ling, H.I.: Framework for limit state design of geosyntheticreinforced walls and slopes. Transport. Infrastruct. Geotechnol. 1(2), 129-164 (2014)

9. Leshchinsky, D., Zhu, F., Meehan, C.L.: Required unfactored strength of geosynthetic in reinforced earth structures. J. Geotech. Geoenviron. Eng. 136(2), 281-289 (2010)

10. Leshchinsky, D., Ebrahimi, S., Vahedifard, F., Zhu, F.: Extension of Mononobe-Okabe approach to unstable slopes. Soils Found. 52(2), 239-256 (2012)

11. Leshchinsky, D., Zhu, F.: Resultant force of lateral earth pressure in unstable slopes. J. Geotech. Geoenviron. Eng. 136(12), 1655-1663 (2010)

12. Vahedifard, F., Leshchinsky, B.A., Sehat, S., Leshchinsky, D.: Impact of cohesion on seismic design of geosynthetic-reinforced earth structures. J. Geotech. Geoenviron. Eng. 140(6), 04014016 (2014)

13. Vahedifard, F., Leshchinsky, D., Meehan, C.L.: Relationship between the seismic coefficient and the unfactored geosynthetic force in reinforced earth structures. J. Geotech. Geoenviron. Eng. 138(10), 12091221 (2012)

14. Duncan, J.M., Wright, S.G.: Soil strength and slope stability, pp. 59-60. Wiley, New Jersey (2005)

15. AASHTO: LRFD bridge design specifications, 6th edn. AASHTO, Washington, DC (2012)

16. National Concrete Masonry Association (NCMA): design manual for segmental retaining walls, 3rd Edn. Herndon, VA (2010) 